\title{
A multicentre single-blind randomized controlled trial comparing bipolar and monopolar transurethral resection of the prostate
}

\author{
Carlos E. Méndez-Probst, MD; Linda Nott, RN; Stephen E. Pautler, BSc, MD, FRCSC; Hassan Razvi, MD, FRCSC
}

Division of Urology, Department of Surgery, The University of Western Ontario, London, ON

See related article on page 390.

Cite as: Can Urol Assoc J 2011;5(6):385-9; hitp://dx.doi.org/10.5489/cuai.10199

\section{Abstract}

Introduction: Monopolar transurethral resection of the prostate (TURP) is the gold standard surgical therapy for men with lower urinary tract symptoms due to benign prostatic hyperplasia. Although generally considered safer, TURP experience is limited in Canada. Methods: Forty-three patients from 5 Canadian centres were randomized to TURP with either bipolar or monopolar platforms. Patients underwent baseline determinations of American Urological Association (AUA) symptom score, peak urinary flow rate, postvoid residual bladder volume and transrectal ultrasound prostate volume. Primary outcome measures were improvement in AUA symptom score, quality of life assessment and bother assessment. Secondary outcomes included procedural times, duration of catheterization, length of hospitalization, complications and the degree of thermal artifact in tissue specimens. Patients were followed for 6 months.

Results: Twenty-two patients were treated with bipolar and 21 with monopolar TURP. Preoperative demographics were not statistically different between groups. Postoperative data collection times were equivalent in AUA symptom, quality of life, bother and sexual function assessments. No differences were observed in the procedure time (60.7 $\mathrm{min}$, bipolar vs. 47.4 , monopolar) or the duration of urethral catheterization (1.5 days, bipolar vs. 1.1, monopolar). More patients in the bipolar group were discharged on the same day of surgery. There were no differences in the degree of tissue thermal artifact or complication rate.

Conclusion: This trial suggests equivalent short-term outcomes for men undergoing monopolar or bipolar TURP.

\section{Introduction}

Benign prostatic hyperplasia $(\mathrm{BPH})$ is a highly prevalent condition in the adult male, with more than $50 \%$ of the males over 60 having histologically proven prostatic hyperplasia and at least half reporting moderate to severe lower urinary tract symptoms (LUTS). ${ }^{1}$ Currently, the gold standard for the surgical treatment of BPH-related LUTS is transurethral resection of the prostate (TURP). ${ }^{1,2}$ Despite its excellent clinical outcomes, monopolar TURP is associated with well-known and potentially serious complications in $0.8 \%$ to $1.4 \%$ of patients. ${ }^{3-5}$ Risks include thermal tissue damage at the site of surgery or at a distant site by faulty patient grounding, peripheral nerve stimulation, the need for a nonconductive irrigant fluid (which in turn can cause dilutional hyponatremia), fluid overload or specific irrigant toxicities, such as hyperammonemia, myocardial damage and transient blindness. ${ }^{3-5}$

One approach to reduce electrosurgery-related complications has been the adoption of bipolar electrosurgery generators and electrodes to transurethral surgery. With bipolar technology, the ability to use $0.9 \%$ normal saline as an irrigant and the physics of electrical current return theoretically reduce the chances of serious complications during TURP. We report the safety and efficacy results of a multicentre single-blind randomized trial comparing bipolar versus monopolar TURP.

\section{Methods}

This study was designed as a multicentered, single-blind, prospective, randomized trial performed at 5 Canadian centres. The study was designed so that each centre would perform an equal number of bipolar and monopolar procedures at study completion. Ethical approval was obtained at each institution. Patients attending the urology clinics at each site were prospectively approached for enrollment if they presented with LUTS and were candidates for surgery. Inclusion criteria were LUTS suggestive of $\mathrm{BPH}$ and with peak urinary flow rates $<12 \mathrm{~mL} / \mathrm{sec}$ and American Urological Association (AUA) symptom scores $>12$. Patients with acute urinary retention were also enrolled. Exclusion criteria were 
previous prostatic surgery (open or transurethral), history of urethral stricture, failure to discontinue alpha-adrenergic blocking agents for at least 14 days prior to surgery, failure to discontinue 5-alpha reductase inhibitor for at least 1 month prior to surgery, patient interest in future fertility, known neurogenic bladder dysfunction, untreated urinary tract infection, American Society of Anesthesiology (ASA) class $>$ III, patients requiring anticoagulation and patients unwilling or unable to comply with the follow-up schedule.

After obtaining informed consent, we instructed patients to complete the following questionnaires: AUA symptom

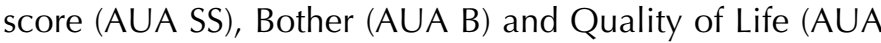
QoL). Blood samples were taken for electrolytes, complete blood count (CBC), blood urea nitrogen (BUN), creatinine $(\mathrm{Cr})$, prostate-specific antigen (PSA), partial thromboplastin time (PTT) and international normalized ratio (INR). Urine for analysis and culture were obtained, on all patients. Peak urinary flow rates (Qmax) and post-void residual (PVR) volumes for those able and not in retention were determined. Physical examination with digital rectal exam, transrectal ultrasound (TRUS) to measure prostate volume and flexible cystoscopy were also performed.

At surgery, patients were randomized $(1: 1)$ to TURP using a 24-26 resectoscope with either a bipolar (VISTA Controlled Tissue Resection System, ACMI, Marlborough, MA) or monopolar (ERBE, Marietta, GA or Valleylab, Tyco Healthcare Group LP, Boulder, CO) energy source. All of the attending urologists before study initiation had performed at least 6 previous bipolar and monopolar TURPs. The surgical technique and antimicrobial prophylaxis regimens were left to each surgeon's preference. Data were recorded for operating room time (defined as time elapsed from the first loop pass to the introduction of the urethral catheter), device failure or intra-operative complications. Resected tissue was sent for pathological review and a pathologist blinded to the treatment assignment reviewed all tissue and provided grading on the degree of thermal artifact based in the percentage of affected tissue (Grade I: 0\%, Grade II: $<25 \%$, Grade III: $25 \%$ to $50 \%$, Grade IV: $50 \%$ to $75 \%$ and Grade V: $>75 \%$ ).

After the procedure, continuous bladder irrigation (CBI) was used until urine output was clear or pink and then discontinued. Post-procedure blood sampling (within 8 hours after TURP) included $\mathrm{CBC}, \mathrm{BUN}, \mathrm{Cr}, \mathrm{HCO}_{3}, \mathrm{Na}, \mathrm{K}$ and $\mathrm{Cl}$. If after 3 hours without $\mathrm{CBI}$ and if the urine was clear and the catheter draining freely, the patient was discharged with the catheter to straight drainage. If no difficulties arose overnight, the catheter was removed the next day in the office. If $\mathrm{CBI}$ was required, patients were admitted to hospital and the catheter was removed when the urine was a light pink colour.

Follow-up visits occurred at 1, 3 and 6 months after surgery. Patients completed AUA Symptom, Bother and Quality of Life questionnaires and peak uroflow and PVR urine were measured. At the 6-month visit, flexible cystoscopy was also performed.

The primary outcome parameter was the comparison of AUA symptom score change (preoperative and postoperative) between monopolar and bipolar TURP. Secondary outcomes included the change in preoperative and postoperative AUA Bother and Quality of Life scores, length of time requiring $\mathrm{CBI}$ and urethral catheterization, length of hospital stay, amount of tissue charring on resected chips and volume of PVR urine. Procedure safety was evaluated by measuring blood loss with hemoglobin levels and transfusion rate. The rate of bladder neck contractures or urethral strictures at 6 months was measured.

Statistical analysis was performed using Student's t-test and Chi-square on a commercial software package (GraphPad Prism 4, GraphPad Software Inc. San Diego, CA). Our sample size calculation suggested that 88 patients per randomization arm were required to demonstrate superiority of one device over the other.

\section{Results}

A total of 43 patients were enrolled in the trial; 22 were randomized to bipolar and 21 to monopolar (Table 1). In both groups age, height, weight, body mass index (BMI), PSA, prostate volume by TRUS, duration of symptoms in years, number of comorbidities, presence of acute urinary retention, volume of PVR urine, maximum Qmax, AUA SS, $A \cup A B$ and $A \cup A$ QoL questionnaires were not statistically different (Table 2).

The mean operative time in the bipolar arm was 60.78 min (range: $15-109$ ) versus 47.48 min (range: 12-95) in the monopolar arm $(p=0.08)$. Anaesthesia type (general vs. spinal), and grams of resected tissue (bipolar 22.5 [range: 2-76] vs. monopolar 19.3 ([range: 7-46.2]) were not statistically different. There were no device failures in either group. No intra-operative complications were encountered.

On histological examination of the surgical specimen there was no difference in the amount of thermal artifact (charring) observed in the resected tissue. One patient in the bipolar group and 2 in the monopolar group had prostate carcinoma foci in the resected chips.

The postoperative decrease in the mean hemoglobin level in the bipolar group of $12.57 \mathrm{mmol} / \mathrm{L}$ was not sig-

\begin{tabular}{lccc}
\hline \multicolumn{3}{l}{ Table 1. Enrollment by centre with randomization } \\
\hline \multirow{2}{*}{ Centres } & \multicolumn{3}{c}{ No. patients } \\
\cline { 2 - 4 } & Bipolar & Monopolar & Total \\
\hline Site 1 & 9 & 9 & 18 \\
Site 2 & 5 & 4 & 9 \\
Site 3 & 5 & 2 & 7 \\
Site 4 & 2 & 4 & 6 \\
Site 5 & 1 & 2 & 3 \\
\hline
\end{tabular}




\begin{tabular}{|c|c|c|c|}
\hline & & Bipolar & Monopolar \\
\hline \multirow{2}{*}{ Age (years) } & Average (SD) & $68(7)$ & $67(7)$ \\
\hline & Range & 51 to 79 & 55 to 85 \\
\hline \multirow{2}{*}{ Height (cm) } & Average (SD) & $176(4)$ & $173(7)$ \\
\hline & Range & 165 to 182 & 155 to 188 \\
\hline \multirow{2}{*}{ Weight (kg) } & Average (SD) & $86(11)$ & $83(10)$ \\
\hline & Range & 64 to 123 & 59 to 110 \\
\hline \multirow{2}{*}{ BMI } & Average (SD) & $28(4)$ & $28(4)$ \\
\hline & Range & 21 to 37 & 21 to 42 \\
\hline \multirow{2}{*}{ PSA } & Average (SD) & $4.52(4.29$ & $4.91(4.26$ \\
\hline & Range & 0.3 to 14.4 & 0.96 to 16.6 \\
\hline \multirow{2}{*}{ Prostate volume (g) by TRUS } & Average (SD) & $57.92(25.56)$ & $50.23(20.74$ \\
\hline & Range & 22.5 to 115 & 20 to 85 \\
\hline \multirow{2}{*}{ Duration of symptoms (years) } & Average (SD) & $9(4)$ & $10(5)$ \\
\hline & Range & 2 to 20 & 1 to 30 \\
\hline Comorbidities & $\mathrm{N}(\%)$ & $18 / 22(81)$ & $16 / 21(76)$ \\
\hline \multirow{3}{*}{$\begin{array}{l}\text { Indwelling Foley catheter } \\
\text { Voided volume }<150 \mathrm{~mL} \\
\text { Voided volume }<125 \mathrm{~mL}\end{array}$} & & 6 & 6 \\
\hline & $\mathrm{N}$ & 18 & 15 \\
\hline & & 10 & 12 \\
\hline \multirow{2}{*}{$\begin{array}{l}\text { PVR urine } \\
\text { By bladder scan }\end{array}$} & Average (SD) & $170.4(197.82)$ & $206.71(211.74)$ \\
\hline & Range & 18 to 656 & 0 to 637 \\
\hline \multirow{2}{*}{ Omax with voided volume $\geq 150 \mathrm{~mL} / \mathrm{sec}$} & Average (SD) & $9.2(2.01)$ & $7.0(2.5)$ \\
\hline & Range & 6 to 11.1 & 2.8 to 10.7 \\
\hline \multirow{2}{*}{ Preoperative AUA Symptom Score } & Average (SD) & $23.2(6.3)$ & $23.4(5.6)$ \\
\hline & Range & 14 to 35 & 13 to 33 \\
\hline \multirow{2}{*}{ Preoperative AUA Bother Score } & Average (SD) & $16.7(6.08)$ & $20.5(5.8)$ \\
\hline & Range & 5 to 28 & 4 to 28 \\
\hline \multirow{2}{*}{ Preoperative AUA Quality of Life Question 4} & Average (SD) & $4.1(1.4)$ & $4.7(0.95)$ \\
\hline & Range & 0 to 6 & 3 to 6 \\
\hline
\end{tabular}

*There were no significant differences when comparing preoperative patient characteristics. SD: standard deviation; BMI: body mass index; PSA: prostate-specific antigen; TRUS: transurethral ultrasound; PVR: post-void residual; AUA: American Urological Association.

nificantly different when compared to $9.10 \mathrm{mmol} / \mathrm{L}$ in the monopolar group. No patient required blood transfusion. The mean change in serum sodium was also not significant. Asymptomatic hyponatremia (serum sodium $<135 \mathrm{mmol} / \mathrm{L}$ ) occurred in 1 patient in the bipolar group and 4 in the monopolar group. No patient developed transurethral resection (TUR) syndrome. No differences were found in mean CBI duration (875.7 min [range: 110-2808) vs. $776.8 \mathrm{~min}$ [range: 118-1950]), mean hospital stay (1.1 [range: 0-3] vs. 1.0 [range: 0-2] days) or catheterization time (1.5 vs. 1.1 days) for bipolar and monopolar groups, respectively. More patients were discharged on the same day of surgery from the bipolar group (7 vs. 3 for the monopolar arm), however this was not statistically significant.

At 1-month follow-up, scores for AUA SS, AUA B, AUA QoL, PVR urine and Qmax were not significantly different between groups. After 1 month, 2 patients in the bipolar group withdrew from the study; 1 patient was diagnosed with prostate cancer and underwent further surgical treatment and another was lost to follow-up for family issues unrelated to the study. At 3 and 6 months, scores for AUA
SS, AUA B, AUA QoL, Qmax and PVR urine were not statistically significant (Table 3 ).

Overall complications occurred in 14 patients in the bipolar group and 9 in the monopolar group (Table 4). Complications requiring intervention were not significantly different between the groups; in the bipolar group, 2 bladder neck contractures $(\mathrm{BNC})$ and one $\mathrm{BNC}$ plus urethral stricture; in the monopolar group, $1 \mathrm{BNC}$ and $2 \mathrm{BNC}$ plus urethral stricture. There were no serious unanticipated adverse events.

Enrollment was suspended as a result of the manufacturer's decision to market a different bipolar platform, leading to an inability to provide loops to the study investigators.

\section{Discussion}

Currently, monopolar TURP is the gold standard for the surgical treatment of BPH-related LUTS. Multiple minimally invasive techniques have been developed in an effort to surpass monopolar TURP, however due to cost, safety profile, learning curve or outcomes none have received widespread acceptance. 


\begin{tabular}{|c|c|c|c|c|c|}
\hline & & & Bipolar & Monopolar & $p$ value \\
\hline \multirow{10}{*}{1 month } & \multirow{2}{*}{ AUA Symptom Score } & Ave (SD) & $9.14(5.87)$ & $9.14(7.2)$ & ns \\
\hline & & Range & 0 to 20 & 0 to 32 & \\
\hline & \multirow{2}{*}{ AUA Bother Score } & Ave (SD) & $6.59(5.23)$ & $6.81(6.79)$ & ns \\
\hline & & Range & 0 to 19 & 0 to 25 & \\
\hline & \multirow{2}{*}{ AUA Quality of Life Score } & Ave (SD) & $4.818(3.647)$ & $4.524(4.082)$ & ns \\
\hline & & Range & 0 to 14 & 0 to 15 & \\
\hline & \multirow{2}{*}{ Omax (voided volume $\geq 150 \mathrm{~mL} / \mathrm{sec}$ ) } & Ave (SD) & $20.66(8.0)$ & $22.35(8.3)$ & ns \\
\hline & & Range & 4.6 to 40.4 & 8.8 to 34.1 & \\
\hline & \multirow{2}{*}{ PVR urine } & Ave (SD) & $106.3(108.6)$ & $90.4(78.4)$ & ns \\
\hline & & Range & 20 to 470 & 15 to 245 & \\
\hline \multirow{10}{*}{3 months } & \multirow{2}{*}{ AUA Symptom Score } & Ave (SD) & $7.29(6.08)$ & $5.5(4.0)$ & ns \\
\hline & & Range & 1 to 19 & 1 to 14 & \\
\hline & \multirow{2}{*}{ AUA Bother Score } & Ave (SD) & $5.4(5.4)$ & $2.8(3.03)$ & ns \\
\hline & & Range & 0 to 14 & 0 to 10 & \\
\hline & \multirow{2}{*}{ AUA Quality of Life Score } & Ave (SD) & $3.476(3.505)$ & $2.714(3.052)$ & ns \\
\hline & & Range & 0 to 11 & 0 to 12 & \\
\hline & \multirow{2}{*}{ Omax (voided volume $\geq 150 \mathrm{~mL} / \mathrm{sec}$ ) } & Ave (SD) & $24.18(10.81)$ & $18.5(8.7)$ & ns \\
\hline & & Range & 8.5 to 47.4 & 7 to 32.9 & \\
\hline & \multirow{2}{*}{ PVR urine } & Ave (SD) & $110.4(91.3)$ & $56.89(57.5)$ & ns \\
\hline & & Range & 8 to 360 & 0 to 220 & \\
\hline \multirow{10}{*}{6 months } & \multirow{2}{*}{ AUA Symptom Score } & Ave (SD) & $6.4(6.2)$ & $5.4(4.1)$ & ns \\
\hline & & Range & 0 to 24 & 1 to 15 & \\
\hline & \multirow{2}{*}{ AUA Bother Score } & Ave (SD) & $3.32(5.26)$ & $3.34(3.5)$ & ns \\
\hline & & Range & 0 to 17 & 0 to 12 & \\
\hline & \multirow{2}{*}{ AUA Quality of Life Score } & Ave (SD) & $2.050(3.034)$ & $2.238(2.508)$ & ns \\
\hline & & Range & 0 to 12 & 0 to 9 & \\
\hline & \multirow{2}{*}{ Qmax (voided volume $\geq 150 \mathrm{~mL} / \mathrm{sec}$ ) } & Ave (SD) & $18.4(8.99)$ & $21.7(10.98)$ & ns \\
\hline & & Range & 6.4 to 40.1 & 5.2 to 43.6 & \\
\hline & \multirow{2}{*}{ PVR urine } & Ave (SD) & $93.7(64.2)$ & $68.9(71.2)$ & ns \\
\hline & & Range & 0 to 269 & 0 to 244 & \\
\hline
\end{tabular}

SD: standard deviation; PVR: post-void residual; AUA: American Urological Association.

The safety of bipolar TURP is enhanced by the use of normal saline eliminating the incidence of the metabolite toxicities and dilutional hyponatremia. This difference could be especially important in teaching institutions, where bipolar TURP could offset the increased risk of TUR syndrome due to the increased resection times usually needed in physician training. ${ }^{6}$ Anecdotally, urologists have observed better intraoperative visibility during bipolar resection, which may also enhance resident education.?

Bipolar TURP provides an equivalent relief of LUTS and QoL improvement at 6 months compared to conventional monopolar TURP. The safety profile of both procedures in regards to operative and postoperative complications was comparable. Bipolar resection was associated with slightly longer resection times than monopolar, with a mean difference of 13 minutes. Whether this finding is related to a learning curve or lack of bipolar experience of the surgeon is difficult to answer. The bipolar resection loop used in this trial was smaller than a standard 24 Fr monopolar loop, per- haps reducing the speed of tissue resection. This observation is supported by the reported calculations of the resection speed of each system $(0.61 \mathrm{~g} / \mathrm{min}$ bipolar vs. $0.74 \mathrm{~g} / \mathrm{min}$ for monopolar). ${ }^{8}$ Our data support this since the initial prostate volume and the amount of resected prostatic tissue were similar among both groups. The impact of bipolar tissue vaporization, which has been shown to be up to $5 \%,{ }^{9}$ had little effect on the resected tissue weight seen in our series.

Recently, Mamoulakis and colleagues calculated that using bipolar TURP in 50 patients will result in one fewer case of TUR syndrome. ${ }^{10}$ One issue raised in the literature is the possibility of an increased incidence of urethral strictures in bipolar TURP patients (6.1\%)., ${ }^{2,7}$ Reports from randomized trials comparing outcomes between monopolar and bipolar TURP, failed to observe higher rates of stricture formation with the bipolar devices. . $^{8,11,12}$

Our mean length of hospital stay although not significantly different between groups (1.091 [range: 0-72 hours] vs. 1.0 days [range: $0-48$ hours] for bipolar and monopolar, 


\begin{tabular}{lcc}
\hline Table 4. Complications & Bipolar & Monopolar \\
\hline & 1 & 0 \\
\hline Urinary tract infection & 3 & 1 \\
Urinary retention & 1 & 1 \\
Dysuria & 0 & 1 \\
Overactive bladder & 0 & 0 \\
TUR syndrome & 1 & 1 \\
Urgency/urge incontinence & 2 & 2 \\
Hematuria requiring intervention & 2 & 1 \\
post discharge & 2 & 2 \\
*Bladder neck contracture & 1 & \\
*Bladder neck contracture plus & & \\
urethral stricture & & \\
\hline
\end{tabular}

*Noted at 6-month follow-up. TUR: transurethral resection.

respectively), compares favourably to other reported series. ${ }^{2}$ There was no difference in the length of indwelling catheter time among groups. This compares favourably with the reported literature, with a range of 23 to 108 hours. $^{2}$ While we report shorter but not significantly different catheter times in the monopolar group, most studies demonstrate the opposite. This difference could be an artifact related to the study protocol. If the patient was discharged on the day of surgery, the catheter was removed in the office the following day (after 8 am) (9 bipolar vs. 3 monopolar). Whereas, if the patient required an overnight hospital stay, the catheter was removed at 6 am (13 bipolar vs. 18 monopolar).

This study's findings are limited by the small sample size. The lack of blinding in the treatment arm to the operating surgeon could also have been a source of bias. However, this may have been partly mitigated by our study design, where raw data analysis was performed by research personnel not involved in data collection. The results are based on a single type of bipolar electrosurgical unit which might not accurately represent or be applicable to other platforms, although data exists that suggest that clinical results are not machine dependent. ${ }^{10}$ The strengths of the trial are that it was a prospective study, treatment allocation was randomized and the patient was blinded to treatment assignment for the duration of the study. It was multicentered to reduce referral and single surgeon bias with the intent that each centre perform similar numbers of each procedure, however with the premature conclusion of the trial, this was not achievable. Consequently, the range of procedures performed at each institution was varied; however, the number of procedures performed by individual surgeons was equivalent regardless of the study site. The study endpoints were standardized across centres and validated instruments were used to measure outcomes.

\section{Conclusion}

Our data demonstrates that at 6 months of follow-up bipolar TURP is equivalent to monopolar in terms of efficacy out- comes as measured by subjective and objective measures. The procedures also have an equivalent complication profile; however, the elimination of a patient return electrode pad and toxicity from hypo-osmolar irrigation fluids may provide an extra level of patient safety. Longer follow-up is needed to determine if this technology will eventually supplant monopolar TURP as the new gold standard.

Acknowledgement: John D. Denstedt in London, Ontario (The University of Western Ontario), Larry Goldenberg in Vancouver, British Columbia and Peter J Pommerville in Victoria, British Columbia (University of British Columbia), Darren T Beiko and James W Wilson in Kingston, Ontario (Queen's University) and Kevin Piercey in Hamilton, Ontario (McMaster University) contributed patients to this study.

Competing interests: The authors received some funding from Olympus/ACMI. This investigator initiated study was supported by ACMI Canada. All sites received financial support and investigative product.

This paper has been peer-reviewed.

\section{References}

1. AUA Practice Guidelines Committee. AUA guideline on management of benign prostatic hyperplasia (2003). Chapter 1: Diagnosis and treatment recommendations. J Urol 2003;170:530-47.

2. Baazeem A, Elhilali MM. Surgical management of benign prostatic hyperplasia: current evidence. Nat Clin Pract Urol 2008:5:540-9.

3. Hawary A, Mukhtar K, Sinclair A, et al. Transurethral resection of the prostate syndrome: almost gone but not forgotten. J Endourol 2009;23:2013-20.

4. Borboroglu PG, Kane $\mathrm{CJ}$, Ward JF, et al. Immediate and postoperative complications of transurethral prostatectomy in the 1990s. J Urol 1999;162:1307-10.

5. Reich 0, Gratzke C, Bachmann A, et al. Morbidity, mortality and early outcome of transurethral resection of the prostate: a prospective multicenter evaluation of 10,654 patients. J Urol 2008; 180:246-9.

6. Gilleran JP, Thaly RK, Chernoff AM. Bipolar PlasmaKinetic transurethral resection of the prostate: reliable training vehicle for today's urology residents. J Endourol 2006;20:683-7.

7. Tefekli A, Muslumanoglu AY, Baykal $M$, et al. A hybrid technique using bipolar energy in transurethral prostate surgery: a prospective, randomized comparison. J Urol 2005;174:1339-43.

8. Singh $H$, Desai MR, Shrivastav $P$, et al. Bipolar versus monopolar transurethral resection of prostate: randomized controlled study. J Endourol 2005; 19:333-8.

9. Starkman JS, Santucci RA. Comparison of bipolar transurethral resection of the prostate with standard transurethral prostatectomy: shorter stay, earlier catheter removal and fewer complications. BJU Int 2005;95:69-71.

10. Mamoulakis C, Ubbink DT, de la Rosette JJ. Bipolar versus monopolar transurethral resection of the prostate: a systematic review and meta-analysis of randomized controlled trials. Eur Urol 2009;56:798-809.

11. Karaman MI, Kaya C, Ozturk M, et al. Comparison of transurethral vaporization using PlasmaKineticJ energy and transurethral resection of prostate: 1-year follow-up. J Endourol 2005; 19:734-7.

12. Hon NHY, Brathwaite D, Hussain $Z$, et al. A prospective, randomized trial comparing conventional transurethral prostate resection with Plasmakinetic 0 vaporization of the prostate: physiological changes, early complications and long-term folllowup. J Urol 2006;176:205-9.

Correspondence: Dr. Hassan Razvi, Urology, St. Joseph's Hospital, 268 Grosvenor St., London, ON N6A 4V2; fax: 519-646-6037; Hassan.Razvi@sihc.london.on.ca 